\title{
Investigation of exchange coupled bilayer Fe/CuMnAs by pump-probe experiment
}

\author{
Vít Saidl ${ }^{1,2}$, Petr Němec ${ }^{1 *}$, Peter Wadley ${ }^{3}$, Kevin W. Edmonds ${ }^{3}$, Richard P. Campion ${ }^{3}$, Vít Novák ${ }^{2}$, Bryan \\ L. Gallagher ${ }^{3}$, František Trojánek ${ }^{1}$ and Tomáš Jungwirth ${ }^{2,3}$
}

${ }^{1}$ Faculty of Mathematics and Physics, Charles University, Ke Karlovu 3, 12116 Prague 2, Czech Republic

2 Institute of Physics ASCR, v.v.i., Cukrovarnická 10, 162 53, Prague 6, Czech Republic

${ }^{3}$ School of Physics and Astronomy, University of Nottingham, Nottingham NG7 2RD, UK

Received ZZZ, revised ZZZ, accepted ZZZ

Published online ZZZ (Dates will be provided by the publisher.)

Keywords Exchange coupling, antiferromagnets, CuMnAs, ultrafast laser spectroscopy

* Corresponding author: e-mail nemec@karlov.mff.cuni.cz

Time-resolved pump-probe magneto-optical method was used to study $\mathrm{Fe} / \mathrm{CuMnAs}$ bilayer. The probe polarization dependence was used to identify and separate parts of detected signals due to Faraday and Voigt magneto-optical effects that provide information about pump-induced magnetization precession in ferromagnetic (FM) $\mathrm{Fe}$ and demagnetization in antiferromagnetic (AF) CuMnAs layers, respectively. We observed a 180 deg phase shift in the precession signal at non-zero magnetic field that we

\begin{abstract}
interpreted as a signature of the magnetic anisotropy induced in the FM by the adjacent AF layer. Unlike in $\mathrm{x}$-ray magnetic linear dichroism experiments, we did not observe any significant reorientation of magnetic moments in CuMnAs by external magnetic field due to the interlayer exchange coupling with Fe. Differences between these two experimental techniques, providing the distinct pictures, are discussed.
\end{abstract}

Copyright line will be provided by the publisher
1 Introduction The exchange interaction between a ferromagnetic (FM) and an antiferromagnetic (AF) layer leads to a unidirectional anisotropy in FM, which is called exchange bias. This phenomenon plays an important role in spin valves where the AF layer fixes the orientation of the FM magnetization [1, 2]. FM/AF bilayers are often studied by static measurements of magnetic hysteresis loops [3-6]. However, dynamical experiments, like the pump and probe technique, can provide a markedly distinct information about the bilayer properties $[4,5,7-10]$.

Compensated AF semimetal CuMnAs [11, 12] is a promising material for AF spintronics [13, 14] where the first realization of an AF memory chip was reported [15]. However, the zero net magnetic moment makes the magnetic characterization of this material a major challenge traditionally, only x-ray magnetic linear dichroism (XMLD) has been used for the determination of the magnetic anisotropy in nanometer-thick AF films [12]. Very recently we have developed a new table-top pump-probe magneto-optical (MO) method that provides the information about magnetic ordering in CuMnAs epilayers [16]. In this paper we apply this MO technique for the study of a $\mathrm{Fe} / \mathrm{CuMnAs}$ bilayer. This bilayer was explored recently by XMLD and it was shown that due to the interlayer ex- change coupling with Fe, the Néel vector in CuMnAs can be rotated by an external magnetic field [17].

\section{Pump-probe experimental study}

2.1 Experimental details We used a Ti:sapphire oscillator (Mai Tai, Spectra Physics, pulse duration 150 fs, repetition rate $80 \mathrm{MHz}$ ) as a source of femtosecond laser pulses with wavelength $920 \mathrm{~nm}$. Each laser pulse was divided into a pump pulse (with fluence $\approx 3 \mathrm{~mJ} \mathrm{~cm}^{-2}$ ) and a probe pulse (fluence $\approx 0.06 \mathrm{~mJ} \mathrm{~cm}^{-2}$ ). The time delay between pulses $(\Delta t)$ was controlled by a delay line and they were focused to the same spot on the sample (spot diameter $\approx 30 \mu \mathrm{m}$ ). The polarization of the pump pulses was circular with the helicity controlled by a quarter-wave plate. For the studied materials, the measured MO data were independent of the pump helicity and, to improve the signalto-noise ratio, we used their average for the data analysis. The probe pulses were linearly polarized with the orientation of the polarization plane controlled by a half-wave plate and described by an angle $\varepsilon$ (see inset in Fig. 2). The sample was mounted in a closed-cycle cryostat in such a way that the external magnetic field was applied at angle $\approx$ $60^{\circ}$ with respect to the [110] crystallographic direction of the GaP substrate. The field dependence of the MO signal 
dynamics was measured from positive to negative fields. Measurements were performed at $15 \mathrm{~K}$ for normal incidence of probe pulses in a transmission geometry.

2.2 Sample growth and characterization The experiments were performed on a $\mathrm{Fe}(2 \mathrm{~nm}) / \mathrm{CuMnAs}$ $(5 \mathrm{~nm})$ sample that was capped with a $2 \mathrm{~nm}$ layer of Al. The sample was grown on a $\mathrm{GaP}(001)$ substrate by molecular beam epitaxy. The crystallographic axes of individual layers are oriented in a such way that $\mathrm{Fe}(001)[110] \|$ CuMnAs(001)[100] \| GaP(001)[110].

The magnetic properties of the sample were characterized by a superconducting quantum interference device (SQUID) at $2 \mathrm{~K}$ - see Fig. 1. The measured loops are centered around zero external magnetic field which indicates a negligible exchange bias in the sample. The coercivity is around $7 \mathrm{mT}$ and there is only a weak dependence on the direction of the field, i.e., there is a rather weak in-plane magnetic anisotropy of $\mathrm{Fe}$.

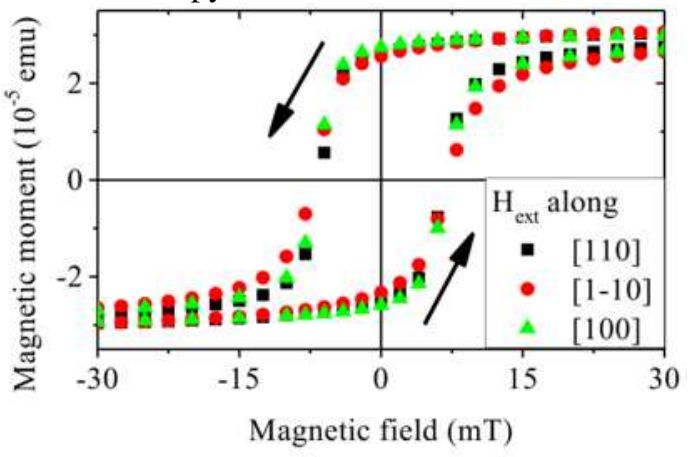

Figure 1 SQUID hysteresis loops measured at $2 \mathrm{~K}$ for various in-plane directions in the sample, which are labelled according to the $\mathrm{GaP}$ substrate directions.

2.3 Results and discussion For fully compensated $\mathrm{AFs}$, the MO signals from oppositely oriented magnetic sublattices cancel for MO effects linear (i.e. odd) in magnetization. On the other hand, MO effects quadratic (even) in magnetization are present also in AFs but it is rather difficult to separate the magnetic order-related signal from other sources of the light polarization change (e.g., strainor crystal structure-related). These problems can be circumvented in a pump-probe experimental scheme where the probe pulse measures a quadratic $\mathrm{MO}$ signal reduction due to the pump-induced demagnetization of the sample [16]. We used the MO Voigt effect, which is closely connected with magnetic linear dichroism (MLD), that is maximal for a normal incidence of probe light on the sample surface [16]. The fingerprint of this MO effect is a harmonic dependence of the change of the polarization rotation (or ellipticity) measured as a function of the mutual orientation of the probe light polarization and magnetic moments, which are described by angles $\varepsilon$ and $\varphi$, respectively (see inset Fig. 2):

$$
M O(\Delta t, \varepsilon)=\frac{2 P}{M} \sin 2(\varphi-\varepsilon) \delta M(\Delta t) .
$$

Here $P$ is the MO coefficient, which scales quadratically with the sublattice magnetization $M$, and $\delta M$ is pumpinduced change of magnetization [16]. In Figs. 2(a) and 2(b) we show MO signals measured in the studied $\mathrm{Fe} / \mathrm{CuMnAs}$ sample for external magnetic fields of 0 and $+670 \mathrm{mT}$, respectively. In addition to the light polarization-sensitive MO signal described by Eq. (1), which is due to the pump-induced demagnetization in CuMnAs, there are also oscillations whose frequency depends on the magnetic field and that we interpret as a precession of ferromagnetic moments in Fe. The phase of these oscillations does not depend on the polarization - see the dashed line in Fig. 2(b). This reveals that the precession of $\mathrm{Fe}$ moments was detected due to the Faraday effect in the polar geometry [18]. A separation of MO signals from Fe and CuMnAs can be performed by fitting the measured data by [19]

$M O(\Delta t)=A \cos (2 \pi f \Delta t+\theta) e^{-\frac{\Delta t}{t_{G}}}+C e^{-\frac{\Delta t}{t_{d}}}+D$.

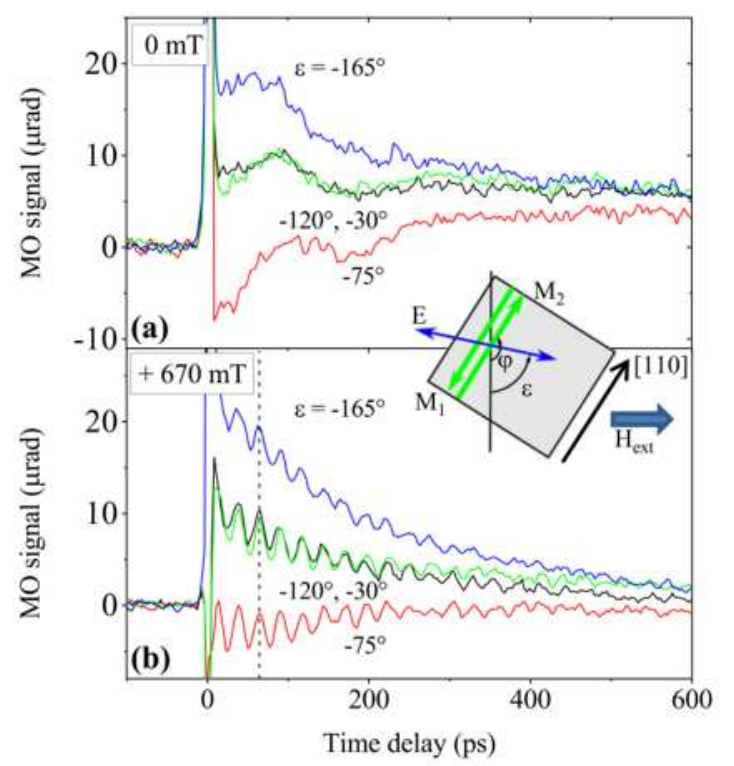

Figure 2 Pump-induced probe polarization rotation measured as a function of time delay in Fe/CuMnAs bilayer for various probe polarization orientations $\varepsilon$ at $0 \mathrm{mT}$ (a) and +670 mT (b). Inset: Schematic depiction of mutual orientations of the crystallographic direction [110] in the GaP substrate, sublattice magnetization $M_{i}$, positive external magnetic field $H_{e x t}$, and probe linear polarization $E$. The dashed vertical line in (b) depicts the independence of the precession phase on $\varepsilon$.

Here $A$ and $C$ are the amplitudes of the precession and demagnetization-related MO signals, respectively, $f$ is the precession frequency, $\theta$ is the precession initial phase, $\tau_{G}$ is the Gilbert damping time, $\tau_{d}$ is the demagnetization decay 
time and $D$ is a polarization-insensitive background, which is coming mainly from the substrate [16].

To study the precession of $\mathrm{Fe}$ we selected the probe polarization of $\varepsilon=-45^{\circ}$ where the non-oscillatory MO signals are suppressed due to the polarization dependence of the Voigt effect (see Fig. 2). Examples of the measured data with fits are shown in Figs. 3(a) and 3(b). In Fig. 3(c) we show the dependence of the precession frequency on the applied magnetic field. The obtained dependence is symmetrical around zero magnetic field. Consistent with the SQUID data, we again see no significant exchange bias which, if present, would shift the position of the lowest frequency to non-zero fields [4]. We fitted the frequency dependence by the Kittel equation [5, 20]

$$
f=\frac{\gamma}{2 \pi} \sqrt{\left(B_{\text {ext }}+B_{A}\right)\left(B_{\text {ext }}+B_{A}+\mu_{0} M_{S}\right)},
$$

where $\gamma$ is the gyromagnetic ratio, $B_{\text {ext }}$ is the external magnetic field, $B_{A}$ is the effective anisotropy field and $M_{S}$ is the saturated magnetization in the ferromagnetic layer. By this procedure we determined that for the studied sample, $B_{A}=$ $16 \mathrm{mT}$ and $M_{S}=1350 \mathrm{emu} \mathrm{cm}{ }^{-3}$, which is in a reasonable agreement with the value of $M_{S}$ expected for Fe [21]. In Fig. 3(d) we show the field dependence of the initial phase of the precession. For large applied external magnetic fields, which considerably exceed the film in-plane anisotropy, the phase differs by $180^{\circ}$ for opposite field directions - see Figs. 3(a) and 3(d). The experimentally measured field dependence of $\theta$ is centered around magnetic field of $-200 \mathrm{mT}$. This behavior we interpret as a signature of CuMnAs-related magnetic anisotropy, which is however not apparent in the SQUID data (see Fig. 1). Such a difference between "dynamic" (i.e., deduced from a pump-probe experiment) and "static" (e.g., deduced from a SQUID data) anisotropy fields was reported also for other FM/AF bilayer systems $[4,5]$. Note that this unidirectional anisotropy is apparent also from the considerable reduction of the precession amplitude observed around -200 $\mathrm{mT}$ [see Fig. 3(d)]. At -92 mT, we even see a time-delayed onset of the oscillations [see Fig. 3(b)]. This we attribute to the change of the magnetic anisotropy due to the pump pulse, which is evolving in time as the local temperature of the excited spot on the sample decreases in time.

The information about CuMnAs layer can be deduced from the polarization-dependent non-oscillatory MO signals which are shown in Fig. 4. Without external magnetic field the fit of the data by Eq. (1) yields $\varphi=149 \pm 2^{\circ}$, which shows that the spin axis in CuMnAs is located along the [110] GaP substrate crystallographic direction (see inset in Fig. 2), in agreement with our previous observations [16]. As apparent in Fig. 4, we have not observed any sizable shift of the Néel vector $\boldsymbol{L}$ in CuMnAs due to the interlayer exchange coupling with $\mathrm{Fe}$ when external magnetic field with a magnitude of $670 \mathrm{mT}$ was applied along the directions $+90^{\circ}$ and $-90^{\circ}$. Similar results were obtained for several temperatures from 15 to $300 \mathrm{~K}$. In the XMLD measurements, on the other hand, a rotation of $\boldsymbol{L}$ by magnetic field was observed in the same sample [17].
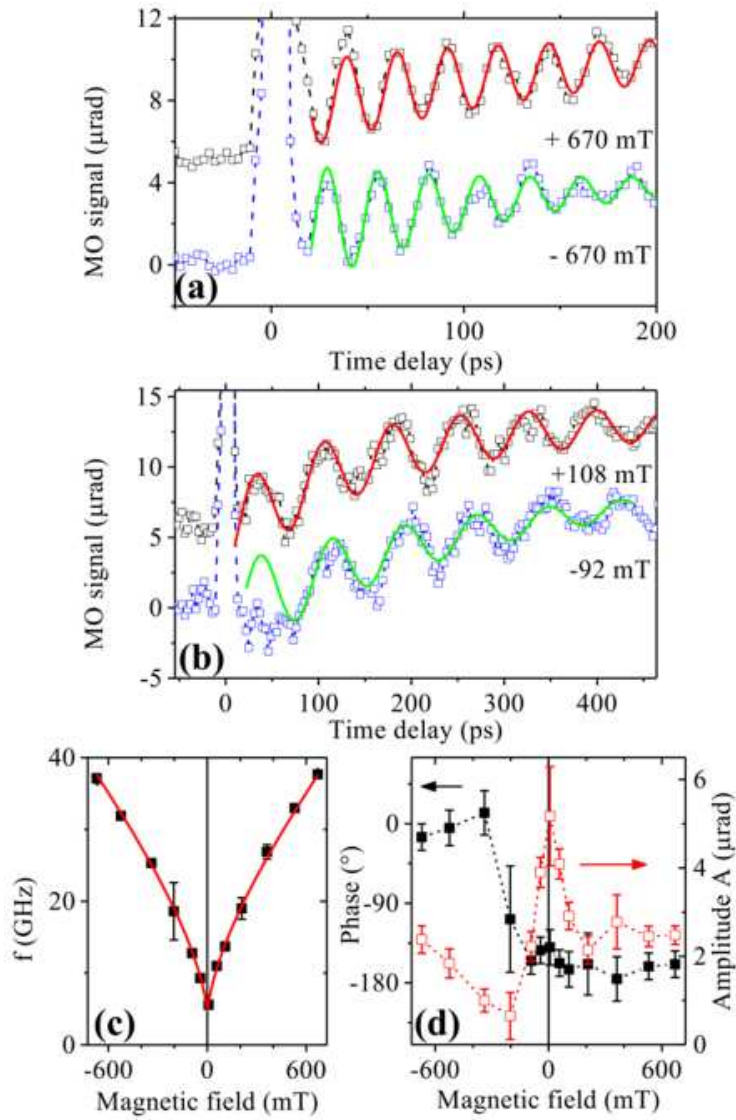

Figure 3 MO signal due to precession of Fe. (a) Timeresolved MO signal measured for $\varepsilon=-45^{\circ}$ at magnetic fields of $+670 \mathrm{mT}$ and $-670 \mathrm{mT}$ (points). Curves are vertically shifted for clarity. (b) Same as (a) for $+108 \mathrm{mT}$ and $-92 \mathrm{mT}$. Solid lines are fits of the measured MO signals by Eq. (2), which were obtained by fitting the experimental data from the second precession maximum and by extrapolating the obtained fit to shorter time delays. (c) Dependence of precession frequency on magnetic field (points). Solid lines are fits by Eq. (3). (d) Dependence of precession phase (solid points) and amplitude (open points) on magnetic field.

One possible explanation of this difference might be a different depth sensitivity of these two techniques: The XMLD experiment was performed in a reflection geometry using a total electron yield that leads to a probing depth of $\approx 3 \mathrm{~nm}$, which is smaller than the $5 \mathrm{~nm}$ CuMnAs film thickness. On the other hand, the optical pump-probe experiment is performed in transmission geometry where the whole material is probed with the same sensitivity. In CuMnAs the uniaxial anisotropy comes from the specific symmetry of bond alignments on the CuMnAs/GaP interface [16] and, therefore, the Fe-mediated rotation of $\boldsymbol{L}$ 
might be more difficult to achieve close to the substrate than at the sample surface. Alternatively, this difference between the static and dynamic experiments might originate from the pump-induced local temperature increase of $\approx 120 \mathrm{~K}$ [16] which is an inherent part of our MO pumpprobe method. It is well-known that the exchange coupling between layers depends strongly on temperature [6]. Consequently, magnetic field can indeed rotate the Néel vector due to the interaction with $\mathrm{Fe}$ in the whole CuMnAs layer but the first pump laser pulse, which heats the sample, might destroy the magnetic alignment of the $\mathrm{Fe}$ and CuMnAs layers. Following this event, the magnetic moments in CuMnAs would rotate towards their easy axis where they would be detected by our stroboscopic method at the original position. Finally, we cannot also exclude a possibility that the sizable pump-induced temperature increase might locally influence the interface - and consequently the exchange coupling - between Fe and CuMnAs layers in a similar way as the sample annealing does [4].

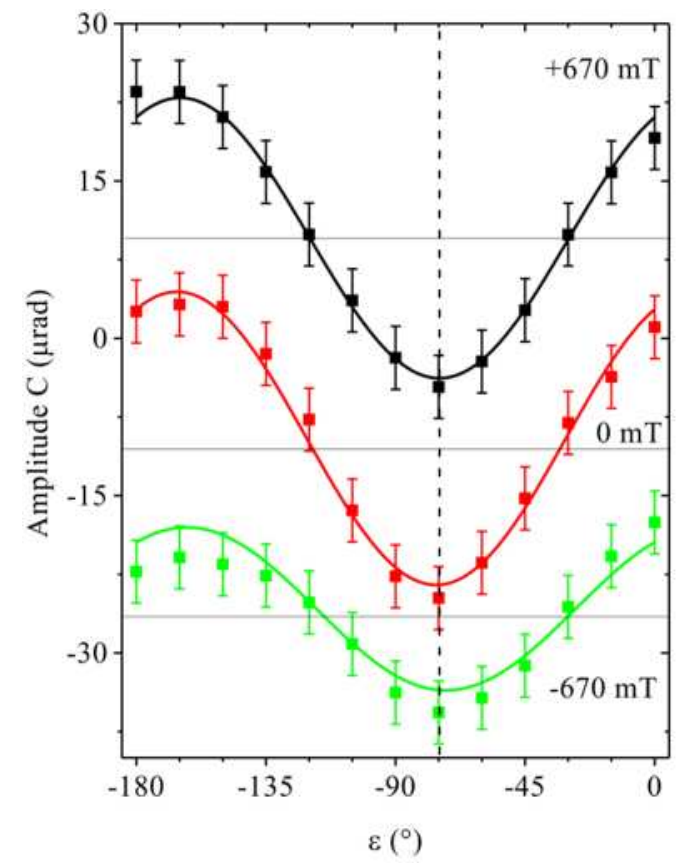

Figure $4 \mathrm{MO}$ signal due to demagnetization in CuMnAs. The amplitude of the demagnetization-related signal $C$ (points), obtained by fitting the measured MO data by Eq. (2), is shown as a function of the probe polarization orientation $\varepsilon$. Data without external magnetic field and for fields $+670 \mathrm{mT}$ and $-670 \mathrm{mT}$, which were applied along the directions $+90^{\circ}$ and $-90^{\circ}$, respectively, were fitted by Eq. (1) and vertically shifted for clarity; the horizontal lines depict the polarization-independent background.

3 Conclusion In conclusion we studied a Fe/CuMnAs bilayer using the time-resolved magnetooptical pump-probe technique. We observed an oscillatory signal due to the precession of Fe moments that is sensitive to the magnetic anisotropy of the Fe layer. By this means we observed a magnetic anisotropy that was not apparent in the SQUID measurements and that we ascribe to interface coupling with the AF. We also discussed possible differences between pump-probe-based MO technique and XMLD that are responsible for the fact that we did not observe any significant reorientation of the magnetic moments in CuMnAs by external magnetic field due to the interlayer exchange coupling with $\mathrm{Fe}$.

Acknowledgements The work was supported by the Grant Agency of the Czech Republic (grant no. 14-37427G), by the Ministry of Education of the Czech Republic (grant no. LM2015087) and by Grant Agency of Charles University (grants nos. 1910214 and SVV-2015-260216). PW acknowledges funding from the University of Nottingham EPSRC Impact Acceleration Account.

\section{References}

[1] R. F. C. Farrow, R. F. Marks, S. Gider, A. C. Marley, S. S. P. Parkin, D. Mauri, J. Appl. Phys. 81, 4986 (1997).

[2] D. H. Han, J. G. Zhu, J. H. Judy, J. M. Sivertsen, J. Appl. Phys. 81, 340 (1997)

[3] J. Nogués, I. K. Schuller, J. Magn. Magn. Mater. 192, 203232 (1999).

[4] J. McCord, R. Kaltofen, T. Gemming, R. Hühne, L. Schultz, Phys. Rev. B 75, 134418 (2007).

[5] F. D. Longa, J. T. Kohlhepp, W. J. M. de Jonge, B. Koopmans, J. Appl. Phys. 103, 07B101 (2008).

[6] X. Martí et al., Phys. Rev. Lett. 108, 017201 (2012).

[7] G. Ju, A. V. Nurmikko, Phys. Rev. B 58, R11857 (1998).

[8] M. Djordjevic, G. Eilers, A. Parge, M. Münzenberg, J. Appl. Phys. 99, 08F308 (2006).

[9] G. Ju, L. Chen, A. V. Nurmikko, Phys. Rev. B 62, 11721177 (2000).

[10] F. D. Longa, J. T. Kohlhepp, W. J. M. de Jonge, B. Koopmans, Phys. Rev. B 81, 094435 (2010).

[11] P. Wadley et al., Nat. Commun. 4, 2322 (2013).

[12] P. Wadley et al., Sci. Rep. 5, 17079 (2015).

[13] E.V. Gomonay, and V.M. Loktev, Low Temp. Phys. 40, 17 (2014).

[14] T. Jungwirth, X. Marti, P. Wadley, and J.Wunderlich, Nature Nanotechnol. 11, 231-241 (2016).

[15] P. Wadley et al., Science 351, 587-590 (2016).

[16] V. Saidl et al., Nat. Photon., in print, arXiv: 1608.01941.

[17] P. Wadley et al., arXiv: 1702.30147

[18] A. K. Zvezdin, A. V. Kotov, Modern Magnetooptics and Magnetooptical Materials (Institute of Physics, 1997), chap. 1 and 3.

[19] N. Tesarova et al., Appl. Phys. Lett. 100, 102403 (2012).

[20] C. Kittel, Introduction to Solid State Physics $-8^{\text {th }}$ edition (Wiley, New York, 2005), p. 381.

[21] Z. Frait, R. Gemperle, Journal de Physique Colloques 32, C1-541-C1-542 (1971) 